\title{
Quantification of volume changes in the descending aorta after frozen elephant trunk procedure using the Thoraflex hybrid prosthesis for type A aortic dissection
}

\author{
Marco Virgilio Usai ${ }^{1 \#}$, Abdulhakim Ibrahim ${ }^{1 \#}$, Alexander Oberhuber ${ }^{1}$, Angelo Maria Dell'Aquila ${ }^{2}$, \\ Sven Martens ${ }^{2}$, Arash Motekallemi ${ }^{2 \#}$, Andreas Rukosujew ${ }^{2 \#}$ \\ ${ }^{1}$ Department of Vascular and Endovascular Surgery, University Hospital Muenster, Muenster, Germany; ${ }^{2}$ Department of Cardiothoracic Surgery, \\ Division of Cardiac Surgery, University Hospital Muenster, Muenster, Germany \\ Contributions: (I) Conception and design: A Rukosujew, A Oberhuber, AM Dell'Aquila, A Motekallemi, A Ibrahim; (II) Administrative support: A \\ Motekallemi, A Rukosujew, MV Usai; (III) Provision of study materials or patients: A Rukosujew, MV Usai, A Ibrahim; (IV) Collection and assembly \\ of data: A Ibrahim, A Motekallemi; (V) Data analysis and interpretation: A Motekallemi, AM Dell'Aquila, A Rukosujew, A Oberhuber, A Ibrahim; (VI) \\ Manuscript writing: All authors; (VII) Final approval of manuscript: All authors. \\ \#These authors contributed equally to this work. \\ Correspondence to: Andreas Rukosujew, MD. Department of Cardiothoracic Surgery, Division of Cardiac Surgery, University Hospital Muenster, \\ Albert-Schweitzer-Campus 1, 48149 Münster, Germany. Email: andreas.rukosujew@ukmuenster.de.
}

Background: Frozen elephant trunk (FET) is an established approach to reduce late complications of type A aortic dissection (AADA) by diminishing false lumen perfusion. Currently, surface size of aortic lumina are evaluated using Computed tomography (CT). However, this $2 \mathrm{D}$ method is prone to error as it evaluates dissection progression slice by slice. Volume measurement on the other hand can overcome this limitation and deliver better insights in aortic remodeling. Therefore, the aim was to quantify volume changes of the descending and abdominal aorta at short- and mid-term follow-up after FET.

Methods: Between April 2015 and March 2018, 20 patients who underwent surgical repair of AADA using the Thoraflex ${ }^{\mathrm{TM}}$ Hybrid Plexus (Vascutek, Terumo Aortic, Scotland) were included in this study. We measured volumetric change before surgical treatment, at discharge, at 12 and at 24 months based on CTAs (Computed tomography angiography). Surfaces and volumes have been analyzed using Aquarius iNtuition (TeraRecon Inc., Foster City, CA, USA).

Results: One hundred fifty-eight volumetric measures were obtained. The findings show a significant increase of volume of the true lumen (TL) while surface measurement of the TL did not show any significant change at other levels besides level C (diaphragm, $\mathrm{P}=0.00193$ ). Variance analysis showed significant increase of volume, whereas no significant change was seen in false lumen. Post-hoc analysis revealed a significance at 24 months $(\mathrm{P}=0.047)$.

Conclusions: Although previous studies outline the clinical benefit of Thoraflex hybrid prosthesis on short-term follow up, this study provides a more precise understanding of aortic remodeling based on volumetric measurement. Thus, quantification of volume changes should be included for the assessment of optimal follow-up timing and consecutive procedure planning.

Keywords: Aortic dissection; hybrid prosthesis; aortic remodeling

Submitted Jul 02, 2020. Accepted for publication Oct 22, 2020.

doi: $10.21037 /$ jtd-20-2356

View this article at: http://dx.doi.org/10.21037/jtd-20-2356 


\section{Introduction}

Late complications after surgical repair of type A aortic dissection, involving the arch and the descending aorta (AADA) due to persistent false lumen patency remain important setbacks (1).

In this setting, recent advances in open and endovascular surgery and an increase in one-stage procedures, which enable an extended repair beyond the proximal aortic arch (2-4), claim to reduce aneurysmal degeneration of the downstream aorta and therefore improve patient outcome. Consequently, these approaches allow to reduce perfusion of false lumen (FL) while providing re-expansion of the distal true lumen (TL) (5).

A well-established surgical approach of AADA consists of a total aortic arch replacement using the frozen elephant trunk (FET) (6-8). This approach aims to reduce aforementioned late complications by decreasing the FL perfusion as the positive remodeling effect of the FET technique not only affects the stentgrafted segment but also the downstream aorta. $w$ results in terms of reduced perioperative mortality, freedom from aortic reoperation and recurrent nerve palsy have been published $(8,9)$. On the other hand, the positive effect on the thoraco-abdominal aorta seems to fade with increasing distance from the (FET-) stentgraft resulting in a gradual narrowing of the true lumen (9).

Currently, size of true and false lumen after FET are being evaluated using CT and less frequently ultrasound. However, diameter measurement alone fails to evaluate the volumetric progression of the dissected aortic lumina adequately, as measurement is based on each slice rather than total volume. Moreover, this method is prone to error as surface measurement relies on exact matching of the same slice over time, which can be difficult in sickle-shaped true lumen. Volume measurement on the other hand can overcome this limitation and delivers better insights in aortic remodeling. Thus, quantification of volume changes instead of surface measurements adds crucial insights in aortic remodeling. This could help optimize follow-up timing and subsequent procedure planning $(9,10)$. However, limited data are available regarding actual volumetric changes of true and false lumen after FET procedure. Especially with regard to consecutive procedures after AADA a better understanding of true and false volume remodeling is mandatory.

Therefore, the aim of this study was to quantify volume changes of the descending and abdominal aorta at short- and mid-term follow-up after FET procedure.

We present the following article in accordance with the MDAR reporting checklist (available at http://dx.doi. org/10.21037/jtd-20-2356).

\section{Methods}

The study was approved by the local ethics committee (protocol number 2018-506-f-S). The study was conducted in accordance with the Declaration of Helsinki (as revised in 2013). Due to the retrospective character of the study informed consent was waived by the institutional review board. Between April 2015 and March 2018, a total of 84 patients underwent surgical treatment of AADA. In these patients, 21 underwent surgical repair using the Thoraflex ${ }^{\mathrm{TM}}$ Hybrid Plexus 4 (Vascutek, Terumo Aortic, Scotland). Thirty-day-mortality was $4.8 \%$. Therefore, 20 patients, i.e., 18 male and 2 female patients with a mean age $57 \pm 17$ years old, were included in this study. Based on laboratory-chemical examinations and CT scans there was no sign of malperfusion or organic ischemia in any patients prior to surgery. Surgery was performed at the department of cardiothoracic surgery, division of cardiac surgery at the University Hospital Münster.

\section{Surgical replacement}

Surgery was performed under moderate hypothermic $\left(28^{\circ} \mathrm{C}\right)$ circulatory arrest and selective bilateral antegrade cerebral perfusion. Near-infrared spectroscopy (NIRS) was used to monitor cerebral tissue oxygenation. After median sternotomy extracorporeal circulation was initiated. Myocardial protection was achieved using retrograde cold blood cardioplegia. After distal transection of the aortic arch the Thoraflex ${ }^{\mathrm{TM}}$ Hybrid Plexus (facilitating selective arch vessel reconstruction) was positioned in the descending aorta. Stentgraft size was determined according to the maximal diameter of true lumen. After completion of the distal anastomosis in zone 3, aortic arch vessel reconstruction was performed under reinitialized perfusion of the lower body.

\section{Volume rendering and measurement}

Based on ECG-gated computed tomography angiograms (CTAs) before surgical treatment, at discharge and at one year and two years follow-up volumetric measurements 

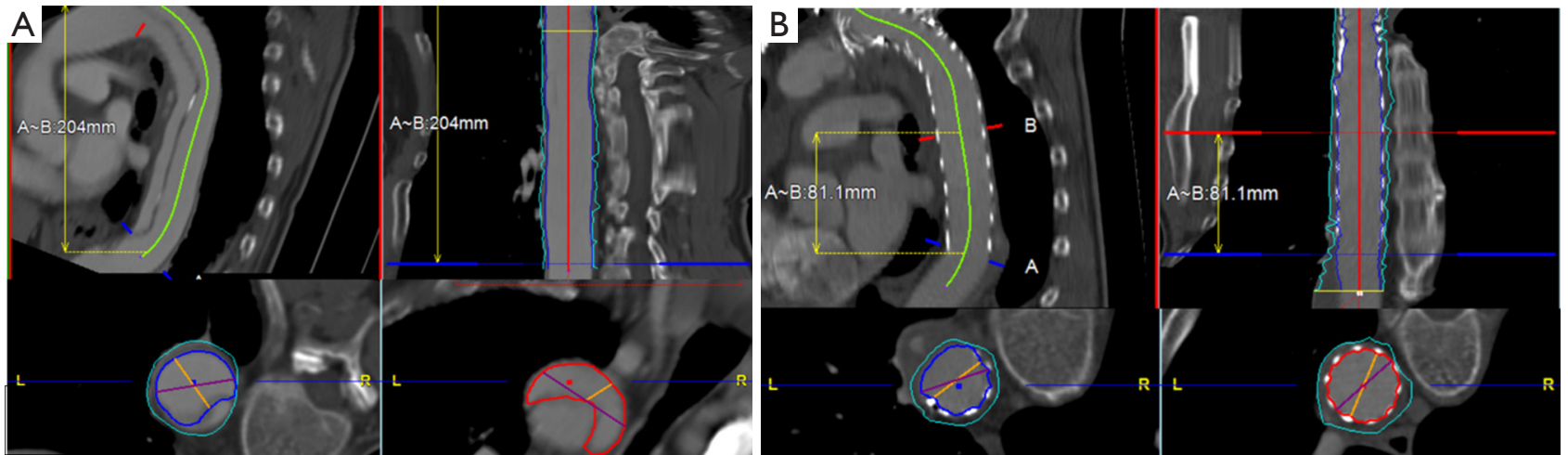

Figure 1 Exemplary CT displaying curved planar and straight multiplanar reconstruction before (A) and 24 months after (B) FET implantation according to the aforementioned stepwise approach using Aquarius iNtuition (TeraRecon Inc., Foster City, CA, USA). Green line: edited center line. Bottom line shows diameter measurement before and after FET implantation.

have been performed. For every patient, we performed a volumetric reconstruction of true, false, and total aortic volume at the different time points. Unfortunately, 9 patients were lost to follow up in the first year and 12 patients in the second year. In case of missing or inconsistent data scans were not included. Overall, we were able to obtain 158 measurements.

Surfaces of the aorta were measured at different levels, i.e., at the left subclavian artery (level A), sixth thoracic vertebral body (level B), diaphragm (level C) and celiac trunk (level D). True lumen volume (TL) and false lumen volume (FL) have been analyzed at the Department of Vascular and Endovascular surgery. First, CT angiography scans were reconstructed automatically with Aquarius iNtuition (TeraRecon Inc., Foster City, CA, USA). Second, after curved planar reformation (CPR), an automatic center line was generated, and then after selection of the preset function for volume measuring, true and false lumen volumes were divided automatically from the software by detecting the level of contrast medium enhancement. Third, the diameter of the true and false lumen was edited manually from the left subclavian artery to the celiac trunk on each slice (slice thickness $1.5 \mathrm{~mm}$ ). All steps of volume rendering were performed by two experienced colleagues of the department of vascular and endovascular surgery with the "four-eyes" principle in order to reduce bias. The true lumen and false lumen were calculated separately for each slice. FL thrombus was included. All volumes are expressed in $\mathrm{cm}^{3}$. Based on previous steps, change in true lumen volume (TLV) and false lumen volume (FLV) was analyzed at the respective time points. Previous volume rendering methods are shown in Figure 1.

\section{Statistical analysis}

All data are expressed as $\mathrm{n}(\%)$ or mean \pm standard deviation. IBM SPSS Statistics for Windows, Version 22 (Armonk, NY, IBM Corp) was used for statistical analysis. The Kolmogorov-Smirnov-test was applied to test the equality of continuous variables for TL and FL at the respective times of measurement (i.e., preoperative, at discharge and 12 and 24 months after discharge). Within the groups (true lumen and false lumen) the one-way analysis of variance (ANOVA) was used to extrapolate significance. Tukey posthoc analysis and Bonferroni correction was applied to compare means at the baseline, discharge and at one and two years follow up. Significance was set at $\mathrm{P}<0.05$.

\section{Results}

One hundred fifty-eight volumetric measures were obtained from the left subclavian artery to the celiac trunk.

Three patients received a secondary aortic procedure due to likely degenerative development of the descending aorta. One patient developed a false lumen aneurysm and received an extension of the stentgraft down to the celiac trunk and occlusion of the false lumen with a vascular plug. A second patient required at the second postoperative day an extension of the thoracic graft due to a collapse of the true lumen and acute kidney injury. The third patient required a prolongation using the Provisional ExTension To Induce COmplete Attachment (PETTICOAT) technique including stenting of the left renal artery due to a collapse of the true lumen and malperfusion. With regard to the initial volume, mean volumetric change in true lumen, false lumen as well 
Table 1 Mean lumen in $\mathrm{cm}^{3}$ at the respective times of measurement

\begin{tabular}{lccccc}
\hline Aortic lumina & Preoperatively $(n=20)$ & At discharge $(n=19)$ & 12 months $(n=11)$ & 24 months $(n=8)$ & Significance \\
\hline Mean TL & 77.03, SD 47.96 & 87.99, SD 33.98 & 113.83, SD 37.33 & 133.84, SD 28.10 \\
Mean FL & 158.33, SD 68.24 & 167.56, SD 90.24 & 164.36, SD 59.72 & 157.20, SD 78.0 & n.s. \\
\hline
\end{tabular}

All data are $\mathrm{cm}^{3}$. SD, standard deviation; TL, true lumen; FL, false lumen; n.s., no statistical significance.

Table 2 Surface measurement of the TL at different levels in $\mathrm{mm}^{3}$

\begin{tabular}{|c|c|c|c|c|c|}
\hline Level & Preoperative & At discharge & 12 months & 24 months & Significance \\
\hline Level B & 315.5, SD 115.7 & 396.5, SD 146.8 & 569.9, SD 187.8 & 616.7, SD 107.5 & n.s. \\
\hline Level C & 294.6, SD 126 & 279.2, SD 132.4 & 340.4, SD 155.4 & 538.7, SD 94.3 & $P=0.00193$ \\
\hline Level D & 291, SD 170.2 & 313.6, SD 147.9 & 563.8, SD 342.8 & 486.7, SD 281.3 & n.s. \\
\hline
\end{tabular}

Surface measurements in $\mathrm{mm}^{3}$ at left subclavian artery (level A), sixth thoracic vertebral body (level B), diaphragm (level C) and celiac trunk (level D). TL, true lumen; n.s., no statistical significance.

as well as surface measurement of the true lumen at abovementioned levels at discharge and mid-term follow up (12 and 24 months) are displayed in Tables 1,2.

The findings show a significant increase in the volume of the true lumen while conventional surface measurement of the true lumen did not show any significant change at level A, B and D. Surface measurement only revealed significant growth at the level of diaphragm (level C) (Figure 2).

Nineteen patients CTAs have been evaluated at time of discharge; 11 patients were available for reevaluation CTA at one year and 8 patients after 2 years follow up. Mean follow up time was 17.05 months. Mean volume of the true lumen (TL), false lumen (FL) and total aortic volume (TAV) before surgery was $77.03 \pm 47.96,158.33 \pm 68.24$, $234.95 \pm 89.72 \mathrm{~cm}^{3}$, respectively. The mean volume of the true lumen grew from 77.03 before surgery to 133.84 at 2 years follow up.

One-way analysis of variance showed significant increase of the true lumen volume, whereas no significant change was seen in false lumen after FET. Post-hoc analysis revealed a significance after 24 months $(\mathrm{P}=0.047)$. However, there was no significant increase in true lumen volume after FET after 12 months ( $\mathrm{P}=0.09)$ (Figure 3).

\section{Discussion}

With the introduction of the hybrid prostheses devices the surgeons' armamentarium evolved significantly in the recent years as it also allows a more adequate treatment of the aortic arch and descending aortic disease. Accordingly, previous studies outlined the benefits of this technique regarding remodeling of true and false lumen (4-9).

Volumetric changes after stentgraft of the descending aorta for type B dissections have been widely described, demonstrating a good long-term behavior of the distal abdominal aorta.

As the benefits of true lumen expansion is selfexplanatory and qualitatively sufficiently outlined, there is no data regarding quantitative volume changes in the descending aorta after FET implantations. As far as we know, this study is the first quantification of volumetric changes of the dissected lumens after surgical repair in AADA using the Thoraflex hybrid prosthesis.

Diameter measurements alone appear not to be sensitive and adequate enough to estimate the progression of the dissected aorta. We observed the same limitations in our surface measures of the true lumen. Instead, volumetric quantification provides a precise dimension of the aortic remodeling. Moreover, these volume measurements overcome some of the limitations of diameter measurements, which may be performed only at a few discrete locations, with limitation on sickleshaped true lumen and with difficult replication of the exact location of different measurements in CTAs taken at different times (11).

As there is no consensus in current guidelines with respect to the aspired volumetric change in the respective aortic segment, these findings could set the ground for an 


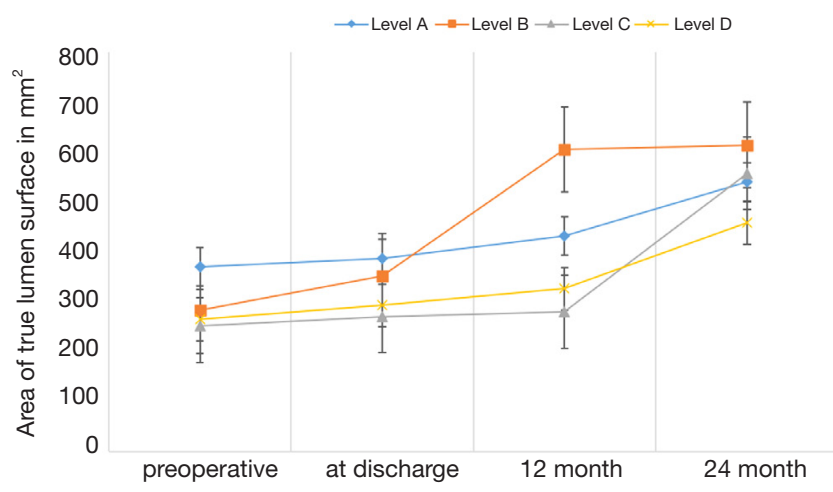

Figure 2 Displaying surface lumina of the TL at different levels. TL, true lumen.

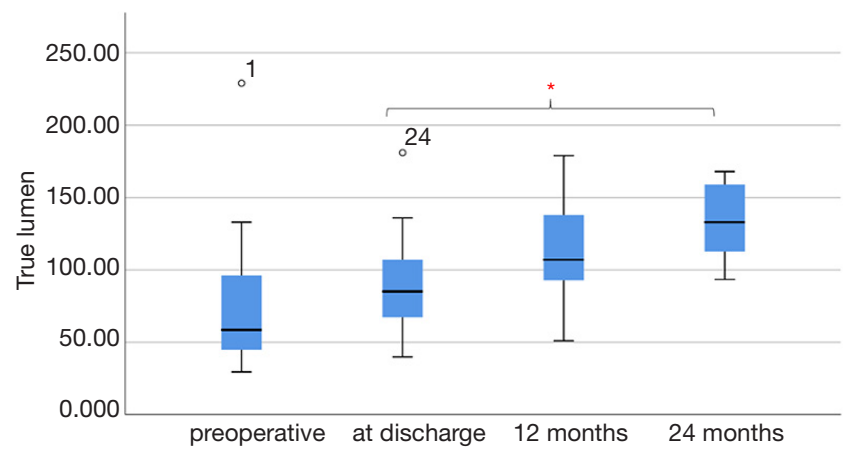

Figure 3 Boxplot of the TL at different time points. *, $\mathrm{P}=0.047$. TL, true lumen.

academic approach to relate quantitative volumetric data to clinical outcome. Consequently, this could potentially lead to improved follow-up pattern.

However, as the current approach was time consuming and prone to individual error, a computed algorithm could facilitate a large-scale evaluation for all cases. Several factors have been recognized to promote false lumen growth. The number of vessels originating from the false lumen has been identified as an independent predictor of false lumen growth. Moreover, increasing age is a negative predictor of aortic growth (12). As the role of false lumen on the aortic growth after type $\mathrm{B}$ dissection (AADB) remains unclear $(13,14)$ incomplete false lumen thrombosis is known as the most predictive for aortic growth in type $\mathrm{B}$ aortic dissection (15).

In AADA, however, patients treated with conventional grafts with re-entries at the level of the supra-aortic branches were prone to unfavorable aortic remodeling (16).
In line with these findings, there are lower rates of mid- to long-term reintervention and false lumen non-thrombosis in patients who underwent a more "complete" aortic repair with hybrid grafts. This may be due to an extended repair of the aorta in the descending tract $(4,5,16)$.

Volumetric change after type B dissection has been thoroughly investigated. Previous studies $(17,18)$ demonstrated that thoracic endografting for acute type $B$ dissections promotes significant thoracic aortic remodeling with true lumen expansion and false lumen regression in the abdominal aorta in early follow up, in particular when adding a bare-metal stent $(17,18)$. Despite these findings, our data did not show false lumen regression after FET implantation. Another study (18) evaluated the long-term effects of the PETTICOAT procedure for acute complicated type B aortic dissection. They confirmed long-term effects of aortic remodeling with TL expansion in the thoracic and abdominal segment while highlighting the positive effect of a complete false lumen obliteration as a major prognostic factor for late complications. In line with these findings, Weiss and colleagues (19) showed that the FET technique for AADA provides a high rate of FL thrombosis in the thoracic aorta compared to conventional methods.

Over all, residual patent false lumen is associated with poor long-term outcome, regardless of dissection type $(20,21)$. The possible role of total aortic coverage using a bare metal stent after aortic arch hybrid procedure simulating a PETTICOAT technique is well evaluated. Although this is an interesting solution, no clinical studies are available evaluating its safety (21).

In our cohort almost all patients showed a significant expansion of true lumen volume, whereas only one patient needed reintervention because of false lumen aneurysm. These results, confirm that this treatment provides a sufficient coverage of the tear zone and possible further entries, which are often culprit for false lumen growth.

This study is limited by the small sample size. The lack of standardized protocols for predetermined follow-up end points might explain the relatively large number of patients lost for mid-term follow-up. This also may have biased our data as follow-up CTAs for 1- and 2-year-endpoints were roughly planed around that time.

However, despite aforementioned limitation, these findings can provide a better understanding of dissection volume and pathology as the dynamic of volume change after FET becomes measurable for the first time.

\section{Conclusions}


In our experience, FET implantation with the Thoraflex hybrid prosthesis for AADA leads to measurable increase of true lumen volume (TL) of the aorta with relatively low rate of false lumen growth. In our preliminary experience, volumetric changes of the aorta seem to be more reliable in detecting remodeling of the aorta. Standardized protocols and a prospective data collection of larger cohorts are needed to confirm this data.

\section{Acknowledgments}

Funding: None.

\section{Footnotes}

Reporting Checklist: The authors have completed the MDAR reporting checklist. Available at http://dx.doi.org/10.21037/ jtd-20-2356

Data Sharing Statement: Available at http://dx.doi. org/10.21037/jtd-20-2356

Peer Review File: Available at http://dx.doi.org/10.21037/jtd20-2356

Conflicts of Interest: All authors have completed the ICMJE uniform disclosure form (available at http://dx.doi. org/10.21037/jtd-20-2356). The authors have no conflicts of interest to declare.

Ethical Statement: The authors are accountable for all aspects of the work in ensuring that questions related to the accuracy or integrity of any part of the work are appropriately investigated and resolved. The study was approved by the local ethics committee (protocol number 2018-506-f-S). The study was conducted in accordance with the Declaration of Helsinki (as revised in 2013). Due to the retrospective character of the study informed consent was waived by the institutional review board.

Open Access Statement: This is an Open Access article distributed in accordance with the Creative Commons Attribution-NonCommercial-NoDerivs 4.0 International License (CC BY-NC-ND 4.0), which permits the noncommercial replication and distribution of the article with the strict proviso that no changes or edits are made and the original work is properly cited (including links to both the formal publication through the relevant DOI and the license). See: https://creativecommons.org/licenses/by-nc-nd/4.0/.

\section{References}

1. Rylski B, Beyersdorf F, Blanke P, et al. Supracoronary ascending aortic replacement in patients with acute aortic dissection type A: what happens to the aortic root in the long run? J Thorac Cardiovasc Surg 2013;146:285-90.

2. Gariboldi V, Grisoli D, Kerbaul F, et al. Long-term outcomes after repaired acute type A aortic dissections. Interact Cardiovasc Thorac Surg 2007;6:47-51.

3. Jakob H, Tsagakis K, Tossios $\mathrm{P}$, et al. Combining classic surgery with descending stent grafting for acute DeBakey type I dissection. Ann Thorac Surg 2008;86:95-101.

4. Takagi H, Umemoto T; ALICE Group. A Meta-Analysis of Total Arch Replacement With Frozen Elephant Trunk in Acute Type A Aortic Dissection. Vasc Endovascular Surg 2016;50:33-46.

5. Shrestha M, Haverich A, Martens A. Total aortic arch replacement with the frozen elephant trunk procedure in acute DeBakey type I aortic dissections. Eur J Cardiothorac Surg 2017;51:i29-34.

6. Shrestha M, Fleissner F, Ius F, et al. Total aortic arch replacement with frozen elephant trunk in acute type A aortic dissections: are we pushing the limits too far? dagger. Eur J Cardiothorac Surg 2015;47:361-6; discussion 366.

7. Karck M, Chavan A, Khaladj N, et al. The frozen elephant trunk technique for the treatment of extensive thoracic aortic aneurysms: operative results and follow-up. Eur J Cardiothorac Surg 2005;28:286-90; discussion 290.

8. Shrestha M, Pichlmaier M, Martens A, et al. Total aortic arch replacement with a novel four-branched frozen elephant trunk graft: first-in-man results. Eur J Cardiothorac Surg 2013;43:406-10.

9. Shrestha M, Kaufeld T, Beckmann E, et al. Total aortic arch replacement with a novel 4-branched frozen elephant trunk prosthesis: Single-center results of the first 100 patients. J Thorac Cardiovasc Surg 2016;152:148-59.e1.

10. Leontyev S, Misfeld M, Daviewala P, et al. Early- and medium-term results after aortic arch replacement with frozen elephant trunk techniques-a single center study. Ann Cardiothorac Surg 2013;2:606-11.

11. Melissano G, Bertoglio L, Rinaldi E, et al. Volume changes in aortic true and false lumen after the "PETTICOAT" procedure for type B aortic dissection. J Vasc Surg 2012;55:641-51.

12. Kamman AV, Brunkwall J, Verhoeven EL, et al. 
Predictors of aortic growth in uncomplicated type $B$ aortic dissection from the Acute Dissection Stent Grafting or Best Medical Treatment (ADSORB) database. J Vasc Surg 2017;65:964-71.e3.

13. Sueyoshi E, Sakamoto I, Uetani M. Growth rate of affected aorta in patients with type B partially closed aortic dissection. Ann Thorac Surg 2009;88:1251-7.

14. Lombardi JV, Cambria RP, Nienaber CA, et al. Aortic remodeling after endovascular treatment of complicated type B aortic dissection with the use of a composite device design. J Vasc Surg 2014;59:1544-54.

15. Trimarchi S, Tolenaar JL, Jonker FH, et al. Importance of false lumen thrombosis in type B aortic dissection prognosis. J Thorac Cardiovasc Surg 2013;145:S208-12.

16. Heo W, Song SW, Lee KH, et al. Surgery for acute Type I aortic dissection without resection of supra-aortic entry sites leads to unfavourable aortic remodelling. Eur J Cardiothorac Surg 2018;54:34-41.

17. Ruddy JM, Reisenman P, Priestley J, et al. Stent graft therapy for false lumen aneurysmal degeneration in

Cite this article as: Usai MV, Ibrahim A, Oberhuber A, Dell'Aquila AM, Martens S, Motekallemi A, Rukosujew A. Quantification of volume changes in the descending aorta after frozen elephant trunk procedure using the Thoraflex hybrid prosthesis for type A aortic dissection. J Thorac Dis 2021;13(1):60-66. doi: 10.21037/jtd-20-2356 established type $\mathrm{B}$ aortic dissection (FADED) results in differential volumetric remodeling of the thoracic versus abdominal aortic segments. Ann Vasc Surg 2014;28:1602-9.

18. Conrad MF, Carvalho S, Ergul E, et al. Late aortic remodeling persists in the stented segment after endovascular repair of acute complicated type B aortic dissection. J Vasc Surg 2015;62:600-5.

19. Weiss G, Santer D, Dumfarth J, et al. Evaluation of the downstream aorta after frozen elephant trunk repair for aortic dissections in terms of diameter and false lumen status. Eur J Cardiothorac Surg 2016;49:118-24.

20. Cheng D, Martin J, Shennib H, et al. Endovascular aortic repair versus open surgical repair for descending thoracic aortic disease a systematic review and meta-analysis of comparative studies. J Am Coll Cardiol 2010;5 5:986-1001.

21. Kotha VK, Pozeg ZI, Herget EJ, et al. Early Results of the PETTICOAT Technique for the Management of Acute Type A Aortic Dissection. Aorta (Stamford) 2017;5:124-8. 